\title{
Profiles
}

\section{Jiaqi Ma, China CDC's Chief Expert of Health Information}

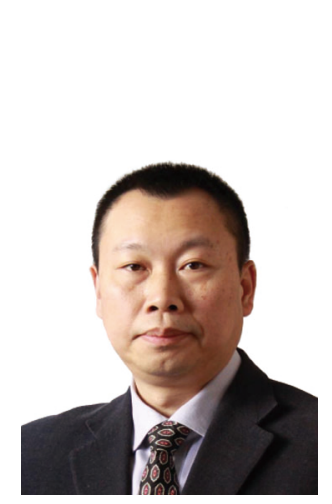

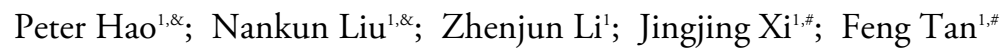

his career, Ma focused on applying information technology for business needs. He learned computer programming languages independently and helped developed of software including the magnetic card health supervision and management information system, iodine deficiency disorders monitoring information system, and infectious disease detection report information system. In 1993, his self-developed "National Endemic Disease Statistical Reporting Software" passed the Ministry of Health (now the National Health Commission) expert review and was applied nationwide. In 1999, as a core technical expert, Ma organized the development of the National Infectious Disease Registration and Report Information System.

In 2002, Ma joined the newly established China CDC. Four months later, the severe acute respiratory syndrome (SARS) epidemic occurred in China, and public health surveillance faced a huge challenge. Ma proposed surveillance system solutions, including promoting online real-time reports, and, within three months, led the establishment of the "Web-based information system for notifiable diseases directed reporting" (predecessor of CISDCP). Under the system, he established a data center for centralized national management. The operation of CISDCP has completely changed the old information management mode of filling out forms and reporting infectious diseases step by step, which significantly improved the timeliness, completeness, and accuracy of infectious disease reporting, and shortened the reporting time of notifiable infectious diseases from 5 days to less than 4 hours. Currently, the system has been operating stably for 16 years and has covered 180,000 users in 73,000 medical institutions across China. It has become a key milestone in China's public health information construction.

From 2004 to 2010, Ma participated in the MBA program of Tsinghua University and the information analysts training at the Chinese Academy of Sciences. He also obtained a master's degree in software engineering from Beihang University. Through years of work and practice, Ma has gradually formed his understanding of the combination of information technology and public health. He believes that information construction must be business-based, requirement-oriented, and technology-led. He has led his team to undertake many research projects under the National Science and Technology Major Project, National Natural Science Foundation of China, and National Science and Technology Support Program. His team published more than 50 papers during the process and obtained 1 patent and 23 software copyrights.

In 2009, China CDC moved to a new location, and Ma led the technical team to start construction on a new information system. His team spent four years establishing the Chinese Data Center for Disease Control and Prevention and the Disaster Recovery Backup Center, thus achieving the transfer and reconstruction of CISDCP. They constructed more than ten core business information systems and the Virtual Private Network (VPN) covering various medical and health institutions at all levels across the country. They also promoted the digital transformation of multiple disease control areas to make the information system safer and more efficient. In 2015, his team was awarded the title of National Advanced Group for Disease Prevention and Control.

In 2017, Ma became the technical director of the construction of the disease prevention and control information system of the National Health Informatization Project, but he did not rest content with old practice. Taking into 
account the advancement of information technology and the increasingly complicated requirements of informatization in the field of disease prevention and control, he proposed and put into practice the information management concept of building an integrated cloud data center and Electronic Disease Record (EDR). Under the guidance of this concept, the reconstructed CISDCP was launched in 2020, which realizes the information management of individual major diseases with life cycle health event monitoring. In the prevention and control of coronavirus disease 2019 (COVID-19), the system quickly made adaptive adjustments, providing functions such as case reporting, epidemiological investigation, transfer treatment, information collection of nucleic acid testing, and big data comparisons to find close contacts.

$\mathrm{Ma}$ is responsible for several academic groups. He initiated the establishment of the Public Health Information Professional Committee of Chinese Medical Information, Big Data Association, and the Professional Committee of Health Big Data and Artificial Intelligence Application of Chinese Prevention Medicine Association and leads the information professionals of the national disease control institutions to carry out scientific research and academic exchanges.

As a Chief Expert of Health Information, Ma always pays attention to the development of information technology and public health informatics. In the future, he will comprehensively promote the development of national public health information through four aspects: 1) by implementing the national information strategy; 2) by guiding and training key technical staff; 3) by strengthening multidisciplinary horizontal cooperation; and 4) by actively participating in the development plan for disease control information. Ma's professional opinions will continue to contribute to the development of public health informatics and public health informatization.

doi: $10.46234 / \mathrm{ccdcw} 2020.203$

\# Corresponding authors: Jingjing Xi, xijj@chinacdc.cn; Feng Tan, tanfeng@chinacdc.cn.

${ }^{1}$ Chinese Center for Disease Control and Prevention, Beijing, China.

\& Joint first authors.

Submitted: September 01, 2020; Accepted: September 15, 2020 